OPEN ACCESS

Edited by:

Sanjay Pandey,

University of Delhi, India

Reviewed by:

Gertrud Tamas,

Semmelweis University, Hungary

Raul Martinez Fernandez,

Centro Integral en Neurociencias A.C.

HM CINAC, Spain

*Correspondence: Jin Woo Chang jchang@yuhs.ac

Specialty section: This article was submitted to Movement Disorders, a section of the journal Frontiers in Neurology

Received: 10 March 2021 Accepted: 03 May 2021

Published: 09 June 2021

Citation:

Kim MJ, Chang KW, Park SH, Chang WS, Jung HH and Chang JW

(2021) Stimulation-Induced Side

Effects of Deep Brain Stimulation in the Ventralis Intermedius and Posterior Subthalamic Area for Essential Tremor.

Front. Neurol. 12:678592.

doi: 10.3389/fneur.2021.678592

\section{Stimulation-Induced Side Effects of Deep Brain Stimulation in the Ventralis Intermedius and Posterior Subthalamic Area for Essential Tremor}

\author{
Myung Ji Kim ${ }^{1}$, Kyung Won Chang ${ }^{2}$, So Hee Park ${ }^{2}$, Won Seok Chang ${ }^{2}$, Hyun Ho Jung ${ }^{2}$ \\ and Jin Woo Chang ${ }^{2 *}$
}

${ }^{1}$ Department of Neurosurgery, Korea University Medical Center, Korea University College of Medicine, Ansan Hospital, Ansan-si, South Korea, ${ }^{2}$ Department of Neurosurgery, Brain Research Institute, Yonsei University College of Medicine, Seoul, South Korea

Deep brain stimulation (DBS) targeting the ventralis intermedius (VIM) nucleus of the thalamus and the posterior subthalamic area (PSA) has been shown to be an effective treatment for essential tremor (ET). The aim of this study was to compare the stimulation-induced side effects of DBS targeting the VIM and PSA using a single electrode. Patients with medication-refractory ET who underwent DBS electrode implantation between July 2011 and October 2020 using a surgical technique that simultaneously targets the VIM and PSA with a single electrode were enrolled in this study. A total of 93 patients with ET who had 115 implanted DBS electrodes (71 unilateral and 22 bilateral) were enrolled. The Clinical Rating Scale for Tremor (CRST) subscores improved from 20.0 preoperatively to 4.3 (78.5\% reduction) at 6 months, 6.3 (68.5\% reduction) at 1 year, and 6.5 (67.5\% reduction) at 2 years postoperation. The best clinical effect was achieved in the PSA at significantly lower stimulation amplitudes. Gait disturbance and clumsiness in the leg was found in 13 patients (14.0\%) upon stimulation of the PSA and in significantly few patients upon stimulation of the VIM $(p=0.0002)$. Fourteen patients $(15.1 \%)$ experienced dysarthria when the VIM was stimulated; this number was significantly more than that with PSA stimulation $(p=0.0233)$. Transient paresthesia occurred in 13 patients $(14.0 \%)$ after PSA stimulation and in six patients (6.5\%) after VIM stimulation. Gait disturbance and dysarthria were significantly more prevalent in patients undergoing bilateral DBS than in those undergoing unilateral DBS $(p=0.00112$ and $p=0.0011$, respectively). Paresthesia resolved either after reducing the amplitude or switching to bipolar stimulation. However, to control gait disturbance and dysarthria, some loss of optimal tremor control was necessary at that particular electrode contact. In the present study, the most common 
stimulation-induced side effect associated with VIM DBS was dysarthria, while that associated with PSA DBS was gait disturbance. Significantly, more side effects were associated with bilateral DBS than with unilateral DBS. Therefore, changing active DBS contacts to simultaneous targeting of the VIM and PSA may be especially helpful for ameliorating stimulation-induced side effects.

Keywords: deep brain stimulation, dysarthria, essential tremor, paresthesia, posterior subthalamic area, stimulation-induced side effect, ventralis intermedius

\section{INTRODUCTION}

Deep brain stimulation (DBS) is a safe and effective treatment for medically refractory essential tremor (ET) (1). The nucleus ventralis intermedius (VIM) of the thalamus has been used as a primary target for DBS (2). However, proximal postural tremors and distal intention tremors are often refractory to VIM DBS. Several studies exploring potential targets for DBS have reported promising results for the posterior subthalamic area (PSA) with respect to tremor suppression (3-10), particularly for tremors that are difficult to control with $\operatorname{VIM} \operatorname{DBS}(5,6)$. The PSA, including the zona incerta, prelemniscal radiation, and cerebellothalamic tract (containing the dentatorubrothalamic tract) $(11,12)$, has been suggested as a potentially effective target for DBS to treat ET. With the advancement of surgical techniques, targeting the PSA by advancing the electrode deeper along the appropriate trajectory from the VIM is now possible $(5,13-15)$. Since the VIM and PSA are located at different contacts along the same electrode, this approach allows for a comparison of the two targets in terms of tremor reduction and stimulationinduced side effects. The investigation of stimulation-induced side effects is necessary, particularly with respect to the PSA, as the destruction of the PSA by lesioning has been associated with significant adverse events (16-18). Therefore, in the present study, we analyzed and compared stimulation-induced side effects and tremor reduction associated with DBS targeting the VIM and PSA via a single electrode based on individual active contacts.

\section{MATERIALS AND METHODS}

\section{Patients}

In the present study, patients with medically refractory ET who were implanted with a single DBS electrode simultaneously targeting the VIM and PSA at our hospital between July 2011 and October 2020 were retrospectively reviewed. Patients who were followed up for $<6$ months and those diagnosed with tremors other than ET (such as dystonia tremor or multiple sclerosis tremor) were excluded. This study received ethical approval from the institutional review board of our institution.

\section{Surgical Procedure}

The surgical technique used in this study has been described previously (13). The operation involved frame-based stereotactic implantation of a DBS electrode that simultaneously targeted the VIM and PSA. Stereotactic $1.5 \mathrm{~T}$ magnetic resonance imaging (MRI) was performed preoperatively, and the data were transferred to the Leksell SurgiPlan (Elekta, Stockholm, Sweden). Standard stereotactic coordinates for VIM localization were as follows: $13-15 \mathrm{~mm}$ lateral to the midline and $25-28.5 \%$ of the length of the anterior commissure-posterior commissure line anterior to the posterior commissure in the intercommissural plane. PSA localization was verified using MRI and the Schaltenbrand atlas. After localizing the targets, the angle of the trajectory necessary to advance the electrode to the PSA between the subthalamic nucleus and the red nucleus was determined using T2-weighted MRI. Trajectory planning was performed using the VIM as the primary target. The coronal and sagittal angles were adjusted as needed to create a trajectory that hit the PSA target, and procedures to evaluate the effect of stimulation on tremors and possible side effects were performed under local anesthesia. During surgery, the ventral thalamic border was identified using microelectrode recordings. The electrode was then advanced to a location that was $5-6 \mathrm{~mm}$ below the ventral thalamic border, and a test stimulation was initiated to evaluate tremor reduction and identify any side effects. Previously, microelectrode recordings have been used to indirectly locate the PSA based on the verification of the motor-evoked firing of VIM neurons and tremor cells inside the VIM nucleus of the thalamus. For permanent stimulation, DBS electrodes (model 3387, Medtronic, Minneapolis, MN, USA) were used. Based on the microelectrode recording results, contacts 0 and 1 were located in the PSA, and contacts 2 and 3 were located in the VIM. After the electrodes were implanted, postoperative computed tomography (CT) was performed before the frame was removed, and the scans were merged with preoperative MR images to determine the positions of the electrodes. Lastly, an implantable pulse generator (Soletra, Activa SC, Activa PC, or Activa RC, Medtronic) was implanted subcutaneously in the infraclavicular region under general anesthesia during the same session.

\section{DBS Contacts and Parameters}

During the first programming session, contact 0 or 1 (PSA) was activated, followed by contact 2 or 3 (VIM) for either single or double monopolar stimulation. In the case of bilateral electrodes, two electrodes were simultaneously activated in the same way. The effect of stimulation on each contact was evaluated to determine stimulation-induced side effects. Lastly, dual activation of contact 0 or 1 and contact 2 or 3 (VIM + PSA) was performed in all patients. The active contacts with the best clinical effects (tremor reduction in the contralateral hand) and the fewest side effects were analyzed. The contacts displaying the best effect were chosen 
for chronic stimulation. The effect of each electrode on the tremors in the contralateral hand was evaluated separately. The amplitude, frequency, and pulse width were modulated using the optimal therapeutic window to improve the tremor. More complex stimulation paradigms, such as interleave or bipolar settings, were chosen if needed. Based on the stimulation parameters required for tremor suppression in each patient, group comparisons were performed. Importantly, even if a surgeon plans a trajectory that hits the PSA and VIM, the final location of the electrode could be altered due to surgical errors, intraoperative adjustments according to microor macrostimulation, and/or trajectory modifications to avoid vessels. The final location of the active contacts that were chosen for chronic stimulation were verified using the postoperative CT scan merged with the preoperative MRI image and the Schaltenbrand atlas using the Leksell SurgiPlan (Figure 1). For further analysis, stereotactic surgical planning was performed using Stealth Station S8 (Medtronic) according to previous target coordinates for each electrode. The planning data were uploaded into SureTune 3 (Medtronic) and merged with the postoperative CT data. Anatomical structures (e.g., VIM, zona incerta, subthalamic nucleus, substantia nigra pars reticulata, and red nucleus) were identified to reveal the relationship between stimulation-induced side effects and the location of the electrodes.

\section{Tremor Outcomes}

Patients were evaluated according to the Clinical Rating Scale for Tremor (CRST) preoperatively and at 6 months, 1 , and 2 years after DBS electrode implantation. As 22 patients received bilateral DBS, the effect of each electrode was evaluated separately. The CRST subscores for the treated upper extremity were calculated by adding the scores of all single items pertaining to that extremity from parts A and B of the CRST (19). The "writing" item was only included for the dominant hand, leading to maximum possible scores of 32 or 28 points per extremity. This evaluation using the CRST was performed according to the methodology presented by Stacy et al. (20).

\section{Statistical Analysis}

Changes in CRST subscores were evaluated using a linear mixed model in the MIXED procedure of SAS (version 9.4, SAS INC., Cary, NC, USA). The analysis used repeated measures data obtained from each patient with no input for missing data because the follow-up period varied for each patient. To determine whether a statistical difference in CRST scores existed between the groups over time, the interaction between group and time was evaluated. In addition, to visualize changes in CRST scores over time, least-square means and standard errors for each time point were obtained to show the mean profile plot. The analysis of variance and Kruskal-Wallis tests were used to compare variables. The Fisher's exact test was used to compare the stimulation-induced side effects of each contact. All $p$-values were two-tailed, and statistical significance was set at $p<0.05$.

\section{RESULTS}

\section{Baseline Characteristics}

A total of 97 patients underwent DBS for ET control between July 2011 and October 2020. Four patients with follow-up periods of $<6$ months, two patients who did not undergo follow-up clinical evaluation after DBS, and one patient who was diagnosed with multiple sclerosis were excluded from this study. Finally, the present study included 93 patients with 115 implanted DBS electrodes (71 unilateral and 22 bilateral). Patient demographics are shown in Table 1. With regard to stimulation parameters, the median amplitude, pulse width, and frequency were $2.4 \mathrm{~V}, 80 \mu \mathrm{s}$, and $160 \mathrm{~Hz}$, respectively.

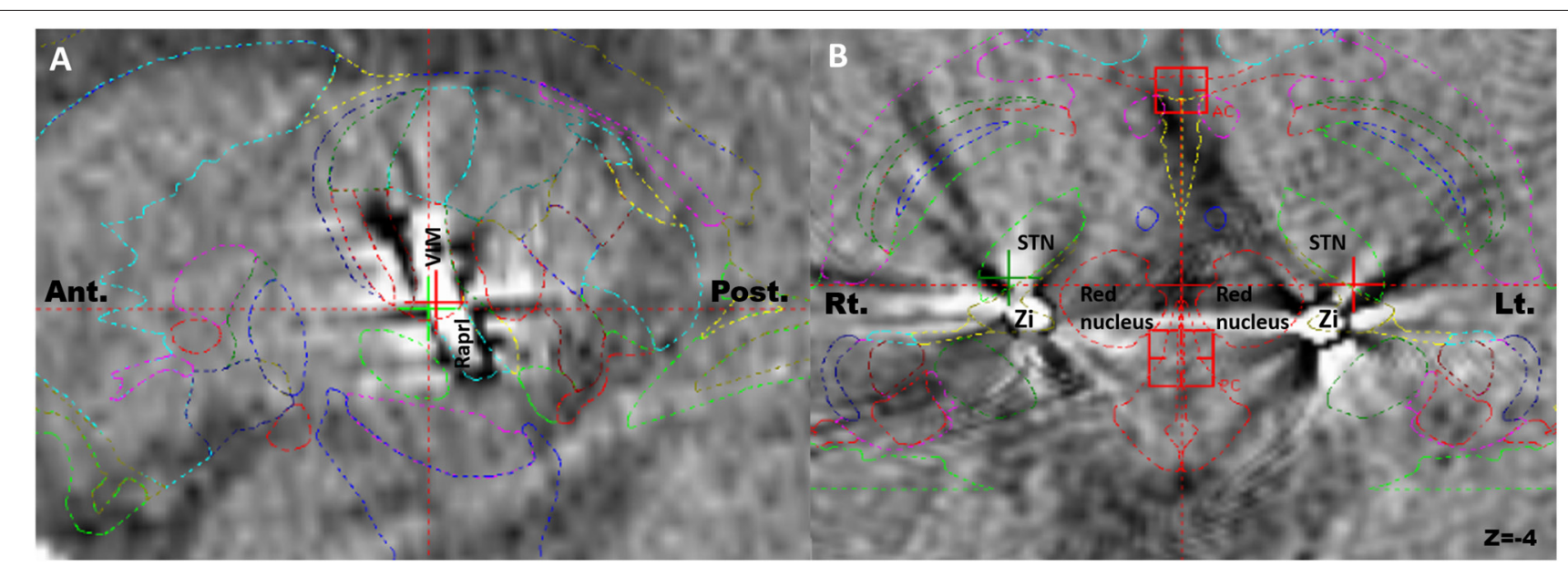

FIGURE 1 | Postoperative computed tomography scans merged with preoperative magnetic resonance images for identification of the actual electrodes and active contacts using Schaltenbrand atlas. (A) The electrode hit the ventralis intermedius and the prelemniscal radiation simultaneously in the sagittal plane. (B) The tips of bilateral electrodes were located in the zona incerta in the axial plane. Ant, anterior; Post, posterior; Rt, right; Lt, left. 


\section{Location of Active Contacts for Chronic Stimulation}

Among the 115 electrodes, 210 active contacts were identified; in $37.8,29.4,25.9$, and $6.9 \%$ of the patients, the contacts 1 , 0,2 , and 3 were chosen for chronic stimulation, respectively (Table 2). Based on the lead analysis, the most stimulated structure was the zona incerta (43.8\%), followed by the VIM $(27.6 \%)$ and the prelemniscal radiation (24.2\%). Notably, our analysis revealed that the surgical procedure had good accuracy in terms of positioning the intended targets to hit the VIM and PSA simultaneously.

\begin{tabular}{lc}
\hline TABLE 1 | Patient demographics and stimulation parameters. & \\
\hline Age $^{\star}$ & $62.9 \pm 7.8$ \\
Follow-up duration $^{\star \star}$ (months) & $38[16,65]$ \\
Sex & \\
Male & $76(78.4 \%)$ \\
Female & $21(21.6 \%)$ \\
Uni/Bilateral*** & \\
Unilateral & $71(73.2 \%)$ \\
Bilateral & $22(22.7 \%)$ \\
Baseline CRST subscore & 19.0 \\
Amplitude $^{\star \star}$ & $2.4[1.9,2.8]$ \\
Pulse width $^{\star *}$ & $80[60,90]$ \\
Frequency $^{\star *}$ & $160[130,160]$ \\
\hline
\end{tabular}

*Values are presented as mean $\pm S D$.

**Values are presented as median [Q1, Q3].

${ }^{* * *}$ Values are presented as percentage.

CRST, Clinical Rating Scale for Tremor.

TABLE 2 | Locations of active contacts for chronic stimulation.

\begin{tabular}{lc}
\hline Electrode & 115 \\
PSA (contact 0 or 1) & $55(47.8 \%)$ \\
VIM (contact 2 or 3) & $9(7.8 \%)$ \\
PSA + VIM (contact 0 or 1 and contact 2 or 3) & $51(44.4 \%)$ \\
Active contacts & 210 \\
0 & $62(29.4 \%)$ \\
1 & $79(37.8 \%)$ \\
2 & $54(25.9 \%)$ \\
3 & $15(6.9 \%)$ \\
Location & \\
Zi & $92(43.8 \%)$ \\
VIM & $58(27.6 \%)$ \\
Raprl & $51(24.2 \%)$ \\
Vop & $6(2.9 \%)$ \\
STN & $2(1.0 \%)$ \\
Voa & $1(0.5 \%)$ \\
\hline PSA &
\end{tabular}

PSA, posterior subthalamic area; Raprl, preleminiscal radiation; STN, subthalamic nucleus; VIM, ventral intermediate nucleus of the thalamus; Voa, ventrooralis anterior nucleus of the thalamus; Vop, ventrooralis posterior nucleus of the thalamus; Zi, zona incerta.

\section{Tremor Reduction and Stimulation Parameters for Chronic Stimulation}

The overall CRST subscore decreased from 20.0 at baseline $(N$ $=115)$ to 4.3 (78.5\% decrease), 6.3 (68.5\% decrease), and 6.5 (67.5\% decrease) at the 6 month $(N=115), 1$ year $(N=$ 93), and 2 year $(N=65)$ follow-ups, respectively (Table 3$)$. A significant difference was observed in CRST subscores over time $(p<0.001)$. The least-square means of the CRST subscores were significantly different among the groups at baseline, with a score of 17.6 in the PSA, 20.7 in the VIM, and 21.7 in the VIM + PSA (Table 3). The CRST subscore decreased from 17.6 to $4.0(77.3 \%$ decrease) in the PSA, from 20.7 to $3.9(81.2 \%$ decrease) in the VIM, and from 21.7 to 5.0 (77.0\% decrease) in the VIM + PSA at the 6 month follow-up. However, the CRST subscore increased slightly after 6 months in all three groups. Although the CRST subscores among the three groups were not statistically significant over time, chronic stimulation 2 years after DBS of the PSA (5.3, 70.0\% decrease from baseline) resulted in slightly better tremor control than that after DBS of the VIM $(6.8,67.1 \%$ decrease from baseline) and the VIM $+\operatorname{PSA}$ (7.7, 64.5\% decrease from baseline). Figure 2 shows the mean profile plot of the changes in CRST subscores over time among the three groups. The mean amplitude, pulse width, and frequency for chronic stimulation were $2.1 \mathrm{~V}, 79.7$ $\mu \mathrm{s}$, and $149.6 \mathrm{~Hz}$, respectively, in the PSA; $3.1 \mathrm{~V}, 81.7 \mu \mathrm{s}$, and $153.3 \mathrm{~Hz}$, respectively, in the VIM; and $2.7 \mathrm{~V}, 87.6 \mu \mathrm{s}$, and $161.3 \mathrm{~Hz}$, respectively, in the VIM + PSA (Table 4). The best clinical effect was achieved with the PSA at significantly lower stimulation amplitudes and frequencies $(p=0.002$ and $p=0.016$, respectively).

\section{Stimulation-Induced Side Effects}

Table 5 shows the stimulation-induced side effects of each contact in the 93 included patients. A total of 13 patients (14.0\%) reported gait disturbance and reduced leg control when either contact 0 or 1 below the intercommissural line (ICL) was stimulated (Figure 3A); this number was significantly higher than that when contact 2 or 3 above the ICL was stimulated $(p=0.0002)$. Dysarthria occurred in 14 patients $(15.1 \%)$ when contact 2 or 3 was stimulated (Figure 3B); this number was significantly higher than that when contact 0 or 1 was stimulated $(p=0.0233)$. Transient paresthesia occurred in 13 patients $(14.0 \%)$ after stimulation below the ICL and in six cases $(6.5 \%)$ after stimulation above the ICL. Gait disturbance and dysarthria occurred significantly more frequently in those undergoing bilateral DBS than in those undergoing unilateral DBS $(31.8 \%$ vs. $8.5 \%, p=0.00112$ and $45.5 \%$ vs. $11.3 \%, p=$ 0.0011 , respectively). Paresthesia resolved either after a reduction in amplitude or change to bipolar stimulation without any loss of optimal tremor control. To reduce the side effect of gait disturbance due to stimulation below the ICL and dysarthria due to stimulation above the ICL, some sacrifice of optimal tremor control was required at that particular electrode contact. These side effects were reversible when changing the active contact to dual VIM + PSA stimulation (Figure 3C) or to bipolar stimulation (Figure 3D). 
TABLE 3 | Clinical Rating Scale for Tremor (CRST) subscores over evaluation visits.

\begin{tabular}{|c|c|c|c|c|c|c|c|c|c|c|}
\hline \multirow[t]{2}{*}{ Follow-up } & \multicolumn{2}{|c|}{ Total $(N=115)$} & \multirow{2}{*}{$\begin{array}{c}p \text {-value } \\
\text { (time) }\end{array}$} & \multicolumn{2}{|c|}{ PSA (contact 0 or 1 ) } & \multicolumn{2}{|c|}{ VIM (contact 2 or 3 ) } & \multicolumn{2}{|c|}{ PSA + VIM (contact 0 or 1 and 2 or 3 ) } & \multirow[t]{2}{*}{$p$-value (group and time) } \\
\hline & $\begin{array}{c}\text { CRST } \\
\text { estimate }\end{array}$ & SE & & $\begin{array}{c}\text { CRST } \\
\text { estimate }\end{array}$ & SE & $\begin{array}{c}\text { CRST } \\
\text { estimate }\end{array}$ & SE & CRST estimate & SE & \\
\hline Baseline & 19.9815 & 0.6426 & - & 17.6306 & 0.8913 & 20.7267 & 2.1853 & 21.6665 & 0.9577 & 0.0095 \\
\hline 6 months & 4.2570 & 0.4380 & $<0.001$ & 4.0150 & 0.6342 & 3.8712 & 1.4714 & 4.9715 & 0.7353 & 0.5914 \\
\hline
\end{tabular}

CRST, Clinical Rating Scale for Tremor; PSA, posterior subthalamic area; SE, standard error; VIM, ventral intermediate nucleus of the thalamus. Boldface type indicated statistical significance $(p<0.05)$. The change in CRST subscores was evaluated using a linear mixed model.

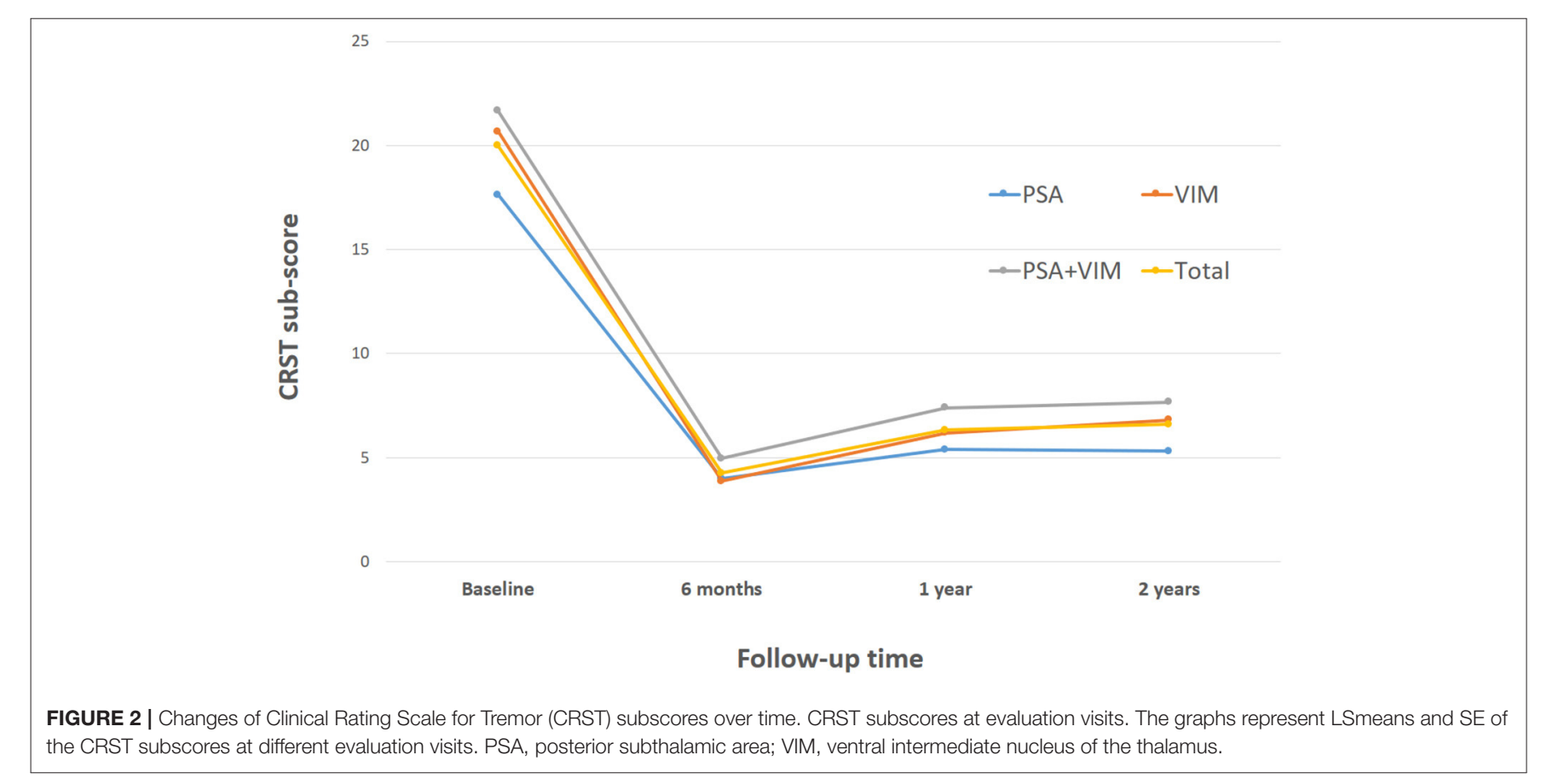

TABLE 4 | Chronic stimulation parameters.

\begin{tabular}{lcccc}
\hline Parameters & PSA $(\boldsymbol{N}=\mathbf{5 5})$ & VIM $(\boldsymbol{N}=\mathbf{9})$ & PSA + VIM $(\boldsymbol{N}=\mathbf{5 1})$ & $\boldsymbol{p}$-value \\
\hline & Mean \pm SD & Mean \pm SD & Mean \pm SD & \\
\hline Voltage & $2.1 \pm 0.8$ & $3.1 \pm 1.8$ & $2.7 \pm 0.7$ & $\mathbf{0 . 0 2 4}$ \\
Pulse width & $79.7 \pm 20.1$ & $81.7 \pm 23.2$ & $87.6 \pm 28.8$ & 0.391 \\
Frequency & $149.6 \pm 16.2$ & $153.3 \pm 19.7$ & $161.3 \pm 16.9$ & $\mathbf{0 . 0 1 6}$ \\
\hline
\end{tabular}

PSA, posterior subthalamic area; VIM, ventral intermediate nucleus of the thalamus. Boldface type indicated statistical significance $(p<0.05)$.

\section{DISCUSSION}

In the present study, we investigated tremor outcomes and stimulation-induced side effects of DBS targeting the VIM and PSA via a single electrode. The strengths of our study are the large number of patients (115 implanted DBS electrodes in 93 patients with ET) and evaluation of long-term outcomes. In the present study, dysarthria, gait disturbance, and paresthesia were the most common stimulation-induced side effects, consistent with previous reports (21-23). We identified that single electrode DBS targeting both the PSA and VIM can be used when stimulationinduced side effects occur.

\section{Clinical Outcomes}

PSA (contact 0 or 1) was most often chosen for chronic stimulation followed by VIM + PSA and VIM (Table 2). Tremor improved from baseline at all time points (Table 3). Additionally, favorable outcomes in terms of overall improvement in CRST subscores for the treated side were observed in this study, and among the three groups, there was no significant difference in tremor suppression with respect to each patient's individual active contacts (Table 3 ). The VIM required a significantly lower stimulation amplitude (Table 4). Additionally, although tremor was less effectively controlled over time with VIM + PSA stimulation (Table 3), the least-square means of CRST subscores were significantly higher at baseline with VIM + PSA 
TABLE 5 | Stimulation-induced side effects on each contact.

\begin{tabular}{|c|c|c|c|c|c|c|}
\hline $\begin{array}{l}\text { Stimulation-induced } \\
\text { side effect }\end{array}$ & $\begin{array}{l}\text { PSA (contact } 0 \\
\text { or 1) } N(\%)\end{array}$ & $\begin{array}{l}\text { VIM (contact } 2 \\
\text { or } 3) \boldsymbol{N}(\%)\end{array}$ & $p$-value & $\begin{array}{l}\text { Unilateral DBS } \\
\text { (total 71) } N(\%)\end{array}$ & $\begin{array}{l}\text { Bilateral DBS } \\
\text { (total 22) } N(\%)\end{array}$ & $p$-value \\
\hline Gait disturbance & 13 (14.0\%) & $0(0 \%)$ & 0.0002 & $6(8.5 \%)$ & 7 (31.8\%) & 0.0112 \\
\hline Dysarthria & $4(4.3 \%)$ & $14(15.1 \%)$ & 0.0233 & $8(11.3 \%)$ & 10 (45.5\%) & 0.0011 \\
\hline Paresthesia & $13(14.0 \%)$ & $6(6.5 \%)$ & 0.1448 & 15 (21.1\%) & $4(18.2 \%)$ & 1.00 \\
\hline
\end{tabular}

Boldface type indicated statistical significance $(p<0.05)$.

DBS, deep brain stimulation; ICL, intercommissural line; PSA, posterior subthalamic area; VIM, ventral intermediate nucleus of the thalamus.

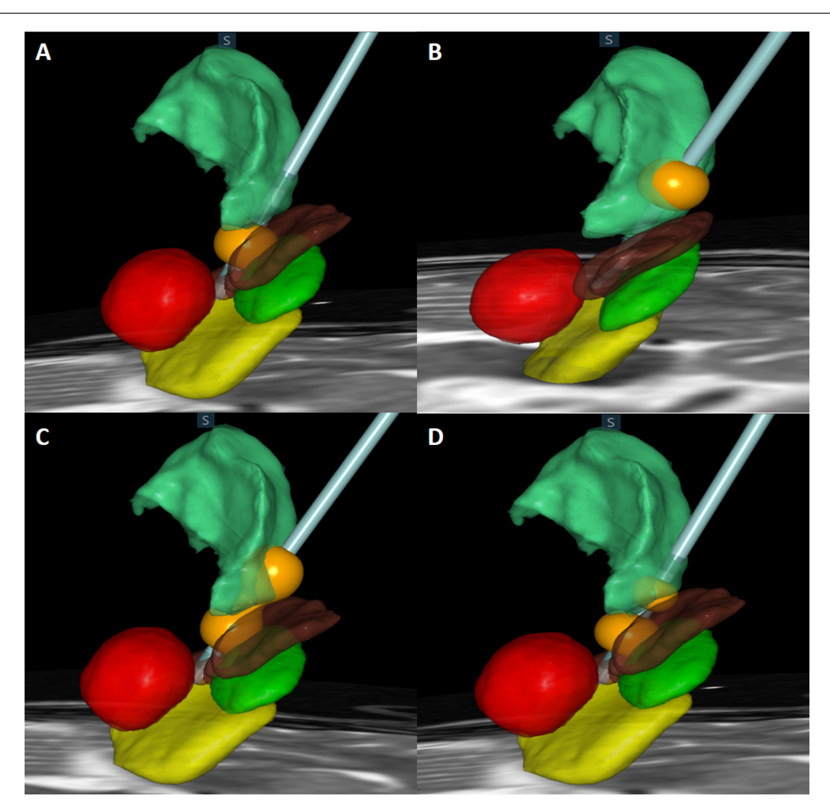

FIGURE 3 | Actual electrode and contact of stimulation in relation to the subthalamic nucleus (STN) (green), substantia nigra (SNr) (yellow), red nucleus (red), zona incerta (Zi) (brown), and ventralis intermedius (VIM) (light green) are shown. (A) Monopolar stimulation of contact 1 located in the Zi. (B) Monopolar stimulation of contact 3 located in the VIM. (C) Dual stimulation of contact 1 in the $\mathrm{Zi}$ and contact 3 in the VIM. (D) Bipolar stimulation of contacts 1 and 2.

stimulation. These findings suggest that the VIM and PSA should be chosen as the active contacts for patients with severe tremors.

\section{Dysarthria}

The most common stimulation-induced side effect associated with VIM DBS was dysarthria (Table 5). A meta-analysis reported that the most commonly reported speech disorder following thalamic DBS was dysarthria (24.2\%) (24). Dysarthria has been frequently observed after $\operatorname{VIM} \operatorname{DBS}(18,25,26)$, most likely due to its effects on the corticobulbar fibers of the internal capsule (27-31). The unintended lateral spread of current can also activate the corticospinal tract and subsequently lead to involuntary muscle contraction of the arms and/or legs as well as lead to dysarthria $(32,33)$. With VIM DBS, this is often considered a consequence of excessively lateral electrode placement, affecting the internal capsule $(18,34)$. Notably, in the present study, stimulation-induced dysarthria was ameliorated by changing active DBS contacts to either VIM + PSA or bipolar stimulation.

\section{Paresthesia}

Gait disturbance and paresthesia were more commonly associated with stimulation of the PSA (Table 5), and paresthesia was usually transient. However, when paresthesia persisted, it could be eliminated by adjusting DBS parameters, such as a reduction in amplitude or bipolar stimulation. Paresthesia is the most common side effect of stimulation of the medial lemniscus, posterior in the subthalamic area $(31,33)$, and the spread of electric current to the ventral caudal thalamic nucleus, which is posterior to the VIM $(25,30,32)$. Paresthesia exacerbated by the spread of electric current away from the VIM can be ameliorated by a more anterior placement of the electrode within the VIM $(35,36)$. Sensory side effects are often considered susceptible to habituation over time and less prone to impede the treatment results (37). Paresthesia can be overcome with programming adjustments (38). Paresthesia can be diminished by decreasing the amplitude of stimulation since it is voltage dependent. Our findings suggest that a slow, gradual increase in amplitude and the use of bipolar stimulation to minimize the spread of current to the nearby medial lemniscus are effective in ameliorating stimulation-induced paresthesia.

\section{Gait Disturbance}

In 10 patients, stimulation via active contacts in the PSA (0 and 1) was changed to VIM + PSA stimulation due to stimulation-induced gait disturbance and reduced leg control despite the loss of optimal tremor control. Previous studies have also observed side effects of PSA stimulation, which mainly included stimulation-induced gait ataxia and clumsiness of the contralateral lower limb $(18,39)$. Stimulation of the cerebellothalamic tract has also been shown to cause postural instability and gait ataxia. These symptoms can be attributed to chronic VIM/PSA stimulation leading to maladaptive plasticity of different fiber tracts (vestibulocerebellar-thalamic afferents and cerebello-rubrospinal tracts) (26, 40-42). More posterior and medial stimulation could activate the cerebellothalamic tracts, leading to gait disturbance or ataxia $(16,32)$. Cerebellar symptoms, including hypotonia, dysmetria, and gait disturbance or imbalance, were often reported after ablation of the subthalamic dorsal area $(18,43-45)$. Although the destruction of the PSA by lesioning has been reported to be associated with significant adverse events $(43,45)$, in the present study, no severe adverse events were observed during the evaluation of PSA DBS. This may be because while PSA DBS overrides tremor oscillations, it does not interrupt patterns of information related to proprioceptive sensations (46). Previous studies have suggested that large pulse width stimulation might account for 
DBS-induced cerebellar side effects and have recommended short pulse width settings for DBS (47-49). Another important issue is that patients with ET often have concomitant cerebellar ataxia, a phenomenon recently classified as ET plus syndrome $(32,50)$. Baseline ataxia may become more apparent after a successful reduction in tremor through DBS.

\section{Unilateral vs. Bilateral}

Gait disturbance and dysarthria were significantly more frequent in those undergoing bilateral DBS than in those undergoing unilateral DBS (Table 5). Previous studies have also reported that stimulation-induced side effects are more frequently observed after bilateral procedures than after unilateral procedures (18, 51-55), with a 2- to 3-fold higher risk of dysarthria and ataxia associated with bilateral procedures $(24,32,36,52,56)$. However, bilateral stimulation is more effective than unilateral stimulation for treating severe bilateral tremors and tremors combined with midline axial tremors (e.g., head tremors) and voice tremors (57). When treating axial tremors that require bilateral DBS, careful evaluation of long-term benefits and risks that may affect the patient's quality of life is essential, and staged operations should be considered at times. Since we activated bilateral electrodes simultaneously for severe bilateral tremors and axial tremors in this study, it was not possible to determine the effect of each electrode individually. Furthermore, it was difficult to determine each electrode's effect on dysarthria and gait disturbance. Therefore, since numerous stimulationinduced side effects associated with bilateral stimulation have been reported, our center recently changed our protocol so that the electrode on the contralateral side with respect to severe tremors is activated first and the other electrode is activated in a delayed manner.

\section{Limitations}

The current study has several limitations. A major limitation is that it was a retrospective review of a single institution's clinical practice. Randomized controlled trials comparing the VIM and PSA directly through "on-off" stimulation of each contact are necessary to confirm our conclusions. Second, the follow-up period varied for each patient. The CRST score was evaluated preoperatively ( $N=115$ electrodes) and again at 6 months $(N$ $=115)$, 1 year $(N=93)$, and 2 years $(N=65)$ postoperation. To compensate for this weakness, we adopted a linear mixed model. Further investigations with continuous follow-up are necessary to confirm the long-term effects of VIM and PSA DBS on tremor reduction, as well as to assess tolerance. Third, only parts A and B of the CRST, which were objective measurements rated by an experienced examiner, were evaluated. Part $\mathrm{C}$ of the CRST, which includes a patient-reported measurement of functional disability due to the tremor, and the Essential Tremor Questionnaire, assessing quality of life in relation to the tremor, were not included since these were subjective measurements. Further studies are needed to determine functional disability and quality of life in patients with ET undergoing DBS. Lastly, while we identified that more adverse side effects were associated with bilateral stimulation than with unilateral stimulation, we failed to confirm the effect of either electrode individually or according to target as both electrodes were activated simultaneously. Therefore, prospectively designed studies are needed to confirm our conclusions.

\section{CONCLUSION}

In the present study, we analyzed tremor outcomes and stimulation-induced side effects in a large sample of patients (115 DBS electrodes in 93 patients with ET) who underwent DBS targeting both the VIM and PSA using a single electrode. Favorable results in terms of overall tremor improvement, stimulation-induced side effects, and surgical accuracy for the intended targets were observed. Knowing the different stimulation-induced side effects associated with the PSA and VIM and their effects on the results of the treatment is essential. The most common stimulation-induced side effects associated with VIM DBS and PSA DBS were dysarthria and gait disturbance, respectively. These side effects were significantly more common in those undergoing bilateral DBS than in those undergoing unilateral DBS. Additionally, in this study, we found that changing active DBS contacts to simultaneous targeting of the VIM + PSA may be especially helpful for ameliorating stimulation-induced side effects.

\section{DATA AVAILABILITY STATEMENT}

The raw data supporting the conclusions of this article will be made available by the authors, without undue reservation.

\section{ETHICS STATEMENT}

The studies involving human participants were reviewed and approved by Severance Hospital Clinical Trial Institutional Review Board. Written informed consent for participation was not required for this study in accordance with the national legislation and the institutional requirements.

\section{AUTHOR CONTRIBUTIONS}

JC: conceptualization, writing (review and editing), and resources. MK: visualization and roles/writing (original draft). KC: data curation and formal analysis. SP: methodology and software. WC: project administration investigation. HJ: supervision and validation. All authors contributed to the article and approved the submitted version.

\section{FUNDING}

This study was supported by Developing technology for commercialization of SEEG procedures and electrode system and grant of the Korea Health Technology R\&D Project through the Korea Health Industry Development Institute (KHIDI), funded by the Ministry of Health \& Welfare, Republic of Korea (Grant Number: HI19C0060). 


\section{REFERENCES}

1. Flora ED, Perera CL, Cameron AL, Maddern GJ. Deep brain stimulation for essential tremor: a systematic review. Mov Disord. (2010) 25:15509. doi: 10.1002/mds.23195

2. Benabid AL, Pollak P, Louveau A, Henry S, de Rougemont J. Combined (thalamotomy and stimulation) stereotactic surgery of the VIM thalamic nucleus for bilateral Parkinson disease. Appl Neurophysiol. (1987) 50:3446. doi: 10.1159/000100803

3. Herzog J, Hamel W, Wenzelburger R, Pötter M, Pinsker MO, Bartussek J, et al. Kinematic analysis of thalamic versus subthalamic neurostimulation in postural and intention tremor. Brain. (2007) 130:1608-25. doi: 10.1093/brain/awm077

4. Blomstedt P, Sandvik U, Fytagoridis A, Tisch S. The posterior subthalamic area in the treatment of movement disorders: past, present, and future. Neurosurgery. (2009) 64:102938. doi: 10.1227/01.NEU.0000345643.69486.BC

5. Blomstedt P, Fytagoridis A, Tisch S. Deep brain stimulation of the posterior subthalamic area in the treatment of tremor. Acta Neurochir. (2009) 151:316. doi: 10.1007/s00701-008-0163-7

6. Blomstedt P, Sandvik U, Tisch S. Deep brain stimulation in the posterior subthalamic area in the treatment of essential tremor. Mov Disord. (2010) 25:1350-6. doi: 10.1002/mds. 22758

7. Barbe MT, Franklin J, Kraus D, Reker P, Dembek TA, Allert N, et al. Deep brain stimulation of the posterior subthalamic area and the thalamus in patients with essential tremor: study protocol for a randomized controlled pilot trial. Trials. (2016) 17:476. doi: 10.1186/s13063-016-1599-3

8. Barbe MT, Liebhart L, Runge M, Deyng J, Florin E, Wojtecki L, et al. Deep brain stimulation of the ventral intermediate nucleus in patients with essential tremor: stimulation below intercommissural line is more efficient but equally effective as stimulation above. Exp Neurol. (2011) 230:1317. doi: 10.1016/j.expneurol.2011.04.005

9. Fytagoridis A, Sandvik U, Aström M, Bergenheim T, Blomstedt P. Long term follow-up of deep brain stimulation of the caudal zona incerta for essential tremor. J Neurol Neurosurg Psychiatry. (2012) 83:25862. doi: 10.1136/jnnp-2011-300765

10. Fytagoridis A, Astrom M, Samuelsson J, Blomstedt P. Deep brain stimulation of the caudal zona incerta: tremor control in relation to the location of stimulation fields. Stereotact Funct Neurosurg. (2016) 94:36370. doi: $10.1159 / 000448926$

11. Gallay MN, Jeanmonod D, Liu J, Morel A. Human pallidothalamic and cerebellothalamic tracts: anatomical basis for functional stereotactic neurosurgery. Brain Struct Funct. (2008) 212:44363. doi: 10.1007/s00429-007-0170-0

12. Coenen VA, Mädler B, Schiffbauer H, Urbach H, Allert N. Individual fiber anatomy of the subthalamic region revealed with diffusion tensor imaging: a concept to identify the deep brain stimulation target for tremor suppression. Neurosurgery. (2011) 68:1069-75. doi: 10.1227/NEU.0b013e31820a1a20

13. Chang WS, Chung JC, Kim JP, Chang JW. Simultaneous thalamic and posterior subthalamic electrode insertion with single deep brain stimulation electrode for essential tremor. Neuromodulation. (2013) 16:23643. doi: 10.1111/j.1525-1403.2012.00503.x

14. Bot M, van Rootselaar F, Contarino MF, Odekerken V, Dijk J, de Bie R, et al. Deep brain stimulation for essential tremor: aligning thalamic and posterior subthalamic targets in 1 surgical trajectory. Oper Neurosurg. (2018) 15:144-52. doi: 10.1093/ons/opx232

15. Barbe MT, Reker P, Hamacher S, Franklin J, Kraus D, Dembek TA, et al. DBS of the PSA and the VIM in essential tremor: a randomized, double-blind, crossover trial. Neurology. (2018) 91:e543-50. doi: 10.1212/WNL.0000000000005956

16. Murata J, Kitagawa M, Uesugi H, Saito H, Iwasaki Y, Kikuchi S, et al. Electrical stimulation of the posterior subthalamic area for the treatment of intractable proximal tremor. J Neurosurg. (2003) 99:70815. doi: 10.3171/jns.2003.99.4.0708

17. Kitagawa M, Murata J, Kikuchi S, Sawamura Y, Saito H, Sasaki H, et al. Deep brain stimulation of subthalamic area for severe proximal tremor. Neurology. (2000) 55:114-6. doi: 10.1212/WNL.55.1.114
18. Fytagoridis A, Blomstedt P. Complications and side effects of deep brain stimulation in the posterior subthalamic area. Stereotact Funct Neurosurg. (2010) 88:88-93. doi: 10.1159/000271824

19. Federau C, Goubran M, Rosenberg J, Henderson J, Halpern CH, Santini $\mathrm{V}$, et al. Transcranial MRI-guided high-intensity focused ultrasound for treatment of essential tremor: a pilot study on the correlation between lesion size, lesion location, thermal dose, and clinical outcome. J Magn Reson Imaging. (2018) 48:58-65. doi: 10.1002/jmri.25878

20. Stacy MA, Elble RJ, Ondo WG, Wu SC, Hulihan J. Assessment of interrater and intrarater reliability of the Fahn-Tolosa-Marin Tremor Rating Scale in essential tremor. Mov Disord. (2007) 22:833-8. doi: 10.1002/mds.21412

21. Pahwa R, Lyons KE, Wilkinson SB, Simpson RK, Jr., Ondo WG, et al. Longterm evaluation of deep brain stimulation of the thalamus. J Neurosurg. (2006) 104:506-12. doi: 10.3171/jns.2006.104.4.506

22. Putzke JD, Wharen RE, Jr., Obwegeser AA, Wszolek ZK, Lucas JA, et al. Thalamic deep brain stimulation for essential tremor: recommendations for long-term outcome analysis. Can J Neurol Sci. (2004) 31:33342. doi: 10.1017/S0317167100003413

23. Lu G, Luo L, Liu M, Zheng Z, Zhang B, Chen X, et al. Outcomes and adverse effects of deep brain stimulation on the ventral intermediate nucleus in patients with essential tremor. Neural Plast. (2020) 2020:2486065. doi: 10.1155/2020/2486065

24. Alomar S, King NK, Tam J, Bari AA, Hamani C, Lozano AM. Speech and language adverse effects after thalamotomy and deep brain stimulation in patients with movement disorders: a meta-analysis. Mov Disord. (2017) 32:5363. doi: $10.1002 / \mathrm{mds} .26924$

25. Chiu SY, Deeb W, Zeilman P, Ramirez-Zamora A, Patterson A, Patel B, et al. Brain atrophy following deep brain stimulation: management of a moving target. Tremor Other Hyperkinet Mov. (2020) 10:46. doi: 10.5334/tohm.546

26. Bruno S, Nikolov P, Hartmann CJ, Trenado C, Slotty PJ, Vesper J, et al. Directional deep brain stimulation of the thalamic ventral intermediate area for essential tremor increases therapeutic window. Neuromodulation. (2020) 24:343-52. doi: 10.1111/ner.13234

27. Bell DS. Speech functions of the thalamus inferred from the effects of thalamotomy. Brain. (1968) 91:619-38. doi: 10.1093/brain/91.4.619

28. Ojemann GA, Ward AA Jr. Speech representation in ventrolateral thalamus. Brain. (1971) 94:669-80. doi: 10.1093/brain/94.4.669

29. Schuurman PR, Bruins J, Merkus MP, Bosch DA, Speelman JD. A comparison of neuropsychological effects of thalamotomy and thalamic stimulation. Neurology. (2002) 59:1232-9. doi: 10.1212/01.WNL.0000031425.37014.55

30. Benabid AL, Pollak P, Gao D, Hoffmann D, Limousin P, Gay E, et al. Chronic electrical stimulation of the ventralis intermedius nucleus of the thalamus as a treatment of movement disorders. J Neurosurg. (1996) 84:20314. doi: 10.3171/jns.1996.84.2.0203

31. Matias CM, Mehanna R, Cooper SE, Amit A, Lempka SF, Silva D, et al. Correlation among anatomic landmarks, location of subthalamic deep brain stimulation electrodes, stimulation parameters, and side effects during programming monopolar review. Neurosurgery. (2015) 11(Suppl. 2):99108. doi: 10.1227/NEU.0000000000000645

32. Roque DA. Segmented versus nonsegmented deep-brain stimulation for essential tremor differ in ataxic side effects. Tremor Other Hyperkinet Mov. (2019) 9:621. doi: 10.5334/tohm.493

33. Pollak P, Krack P, Fraix V, Mendes A, Moro E, Chabardes S, et al. Intraoperative micro- and macrostimulation of the subthalamic nucleus in Parkinson's disease. Mov Disord. (2002) 17(Suppl. 3):S155-61. doi: 10.1002/mds. 10158

34. Deuschl G, Herzog J, Kleiner-Fisman G, Kubu C, Lozano AM, Lyons KE, et al. Deep brain stimulation: postoperative issues. Mov Disord. (2006) 21(Suppl. 14):S219-37. doi: 10.1002/mds.20957

35. Sandoe C, Krishna V, Basha D, Sammartino F, Tatsch J, Picillo M, et al. Predictors of deep brain stimulation outcome in tremor patients. Brain Stimul. (2018) 11:592-9. doi: 10.1016/j.brs.2017.12.014

36. Fasano A, Helmich RC. Tremor habituation to deep brain stimulation: underlying mechanisms and solutions. Mov Disord. (2019) 34:176173. doi: $10.1002 / \mathrm{mds} .27821$

37. Tommasi G, Krack P, Fraix V, Le Bas JF, Chabardes S, Benabid AL, et al. Pyramidal tract side effects induced by deep brain stimulation of 
the subthalamic nucleus. J Neurol Neurosurg Psychiatry. (2008) 79:8139. doi: 10.1136/jnnp.2007.117507

38. Zarzycki MZ, Domitrz I. Stimulation-induced side effects after deep brain stimulation - a systematic review. Acta Neuropsychiatr. (2020) 32:5764. doi: 10.1017/neu.2019.35

39. Fytagoridis A, Astrom M, Wardell K, Blomstedt P. Stimulationinduced side effects in the posterior subthalamic area: distribution, characteristics and visualization. Clin Neurol Neurosurg. (2013) 115:65-71. doi: 10.1016/j.clineuro.2012.04.015

40. Elble RJ. Mechanisms of deep brain stimulation for essential tremor. Brain. (2014) 137:4-6. doi: 10.1093/brain/awt347

41. Groppa S, Herzog J, Falk D, Riedel C, Deuschl G, Volkmann J. Physiological and anatomical decomposition of subthalamic neurostimulation effects in essential tremor. Brain. (2014) 137:109-21. doi: 10.1093/brain/awt304

42. Pollo C, Kaelin-Lang A, Oertel MF, Stieglitz L, Taub E, Fuhr P, et al. Directional deep brain stimulation: an intraoperative double-blind pilot study. Brain. (2014) 137:2015-26. doi: 10.1093/brain/awu102

43. Yasui N, Narabayashi H, Kondo T, Ohye C. Slight cerebellar signs in stereotactic thalamotomy and subthalamotomy for parkinsonism. Appl Neurophysiol. (1976) 39:315-20. doi: 10.1159/000102510

44. Mundinger F, Riechert T. Indication and long-term results with 1400 uni- and bilateral stereotaxic operations in Parkinson's syndrome. Wien Z Nervenheilkd Grenzgeb. (1966) 23:147-77.

45. Blacker HM, Bertrand C, Martinez N, Hardy J, Molina-Negro P. Hypotonia accompanying the neurosurgical relief of essential tremor. J Nerv Ment Dis. (1968) 147:49-55. doi: 10.1097/00005053-196807000-00006

46. Plaha P, Khan S, Gill SS. Bilateral stimulation of the caudal zona incerta nucleus for tremor control. J Neurol Neurosurg Psychiatry. (2008) 79:50413. doi: 10.1136/jnnp.2006.112334

47. Choe CU, Hidding $U$, Schaper M, Gulberti A, Köppen J, Buhmann C, et al. Thalamic short pulse stimulation diminishes adverse effects in essential tremor patients. Neurology. (2018) 91:e704-13. doi: 10.1212/WNL.0000000000006033

48. Kroneberg D, Ewert S, Meyer AC, Kühn AA. Shorter pulse width reduces gait disturbances following deep brain stimulation for essential tremor. $J$ Neurol Neurosurg Psychiatry. (2019) 90:1046-50. doi: 10.1136/jnnp-2018319427

49. Wirth T, Dayal V, de Roquemaurel A, Ferreira F, Vijiaratnam N, Akram H, et al. Endurance of short pulse width thalamic stimulation efficacy in intention tremor. Stereotact Funct Neurosurg. (2020) 1-6. doi: 10.1159/000511716. [Epub ahead of print].

50. Louis ED. The evolving definition of essential tremor: what are we dealing with? Parkinsonism Relat Disord. (2018) 46(Suppl. 1):S87-91. doi: 10.1016/j.parkreldis.2017.07.004

51. Sun X, Huang L, Pan Y, Zhang C, Wang T, Li H, et al. Bilateral posterior subthalamic area deep brain stimulation for essential tremor: a case series. Front Hum Neurosci. (2020) 14:16. doi: 10.3389/fnhum.2020.00016

52. Mitchell KT, Larson P, Starr PA, Okun MS, Wharen RE Jr., et al. Benefits and risks of unilateral and bilateral ventral intermediate nucleus deep brain stimulation for axial essential tremor symptoms. Parkinsonism Relat Disord. (2019) 60:126-32. doi: 10.1016/j.parkreldis.2018.09.004

53. Plaha P, Patel NK, Gill SS. Stimulation of the subthalamic region for essential tremor. J Neurosurg. (2004) 101:48-54. doi: 10.3171/jns.2004.101.1.0048

54. Blomstedt P, Hariz MI. Are complications less common in deep brain stimulation than in ablative procedures for movement disorders? Stereotact Funct Neurosurg. (2006) 84:72-81. doi: 10.1159/000094035

55. Blomstedt P, Hariz GM, Hariz MI, Koskinen LO. Thalamic deep brain stimulation in the treatment of essential tremor: a long-term follow-up. $\mathrm{Br}$ J Neurosurg. (2007) 21:504-9. doi: 10.1080/02688690701552278

56. Peng-Chen Z, Morishita T, Vaillancourt D, Favilla C, Foote KD, Okun MS, et al. Unilateral thalamic deep brain stimulation in essential tremor demonstrates long-term ipsilateral effects. Parkinsonism Relat Disord. (2013) 19:1113-7. doi: 10.1016/j.parkreldis.2013.08.001

57. Obwegeser AA, Uitti RJ, Turk MF, Strongosky AJ, Wharen RE. Thalamic stimulation for the treatment of midline tremors in essential tremor patients. Neurology. (2000) 54:2342-4. doi: 10.1212/WNL.54.12.2342

Conflict of Interest: The authors declare that the research was conducted in the absence of any commercial or financial relationships that could be construed as a potential conflict of interest.

Copyright (c) 2021 Kim, Chang, Park, Chang, Jung and Chang. This is an open-access article distributed under the terms of the Creative Commons Attribution License (CC $B Y)$. The use, distribution or reproduction in other forums is permitted, provided the original author(s) and the copyright owner(s) are credited and that the original publication in this journal is cited, in accordance with accepted academic practice. No use, distribution or reproduction is permitted which does not comply with these terms. 